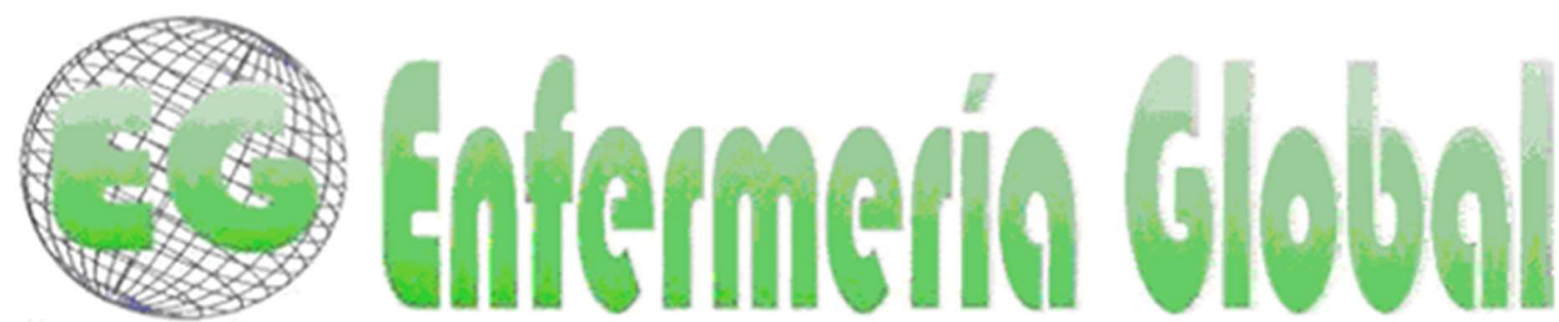

\title{
ORIGINALES
}

\section{Participación de enfermería en Políticas Públicas, ¿Por qué es importante?: Revisión integrativa de la literatura}

Nursing participation in Public Policies, why is it important? Integrative review of the literature

\author{
Ma. Angélica Saldías Fernández ${ }^{1}$ \\ Denisse Parra-Giordano ${ }^{1}$ \\ Trinidad Martí Gutiérrez ${ }^{2}$
}

${ }^{1}$ Departamento de Enfermería Universidad de Chile. Santiago. Chile. drparra@uchile.cl

2 Centro de Investigaciones Biológicas Aplicadas. Chile

\section{https://doi.org/10.6018/eglobal.455361}

Recibido: $10 / 11 / 2020$

Aceptado: 3/03/2021

\section{RESUMEN:}

Objetivo: Analizar la evidencia científica disponible en la literatura en torno al rol de participación política del profesional enfermero.

Método: Revisión integrativa literaria en seis etapas, en bases de datos MEDLINE/PUBMED, ISI WEB OF SCIENCE, EBSCOHOST WEB, LILACS y SCIELO publicados entre 2010 - 2020 en idiomas inglés, portugués y español, ajustada a los requerimientos PRISMA. Los datos fueron resumidos mediante análisis temático.

Resultados: A partir de 75 textos, 23 fueron analizados. Un 35\% de las publicaciones se realizó entre 2010 al 2012, el $48 \%$ son de Estados Unidos, un $65 \%$ se obtuvieron de PUBMED y el $26 \%$ son estudios de caso. Se obtienen dos categorías de trabajo: Participación política del profesional enfermero y Virtudes y competencias. Estrategias y desafíos para participar en PPs. Se muestran similitudes entre ambas categorías.

Conclusión: Enfermería posee activismo sociopolítico impulsado por enfermeras en diferentes contextos. En la medida en que los profesionales de enfermería se hacen conscientes del rol de participación política como una expresión del patrón de conocimiento sociopolítico, su participación favorece el proceso de políticas públicas, y es una oportunidad para el cambio dada su posición estratégica como referentes del cuidado de las personas en los sistemas de salud.

Palabras clave: Enfermería; Política de Salud; Formulación de Políticas.

\section{ABSTRACT:}

Objective: To analyze the scientific evidence available in the literature regarding the role of the political participation of the nursing profession.

Method: Integrative literary review in six stages, in MEDLINE / PUBMED, ISI WEB OF SCIENCE, EBSCOHOST WEB, LILACS, and SCIELO databases 2010 - 2020 in English, Portuguese and Spanish languages, adjusted to PRISMA requirements. The data were summarized by thematic analysis. 
Results: From 75 texts, 23 were analyzed. 35\% of the publications were made between 2010 to 2012 , $48 \%$ are from the United States, $65 \%$ were obtained from PUBMED, and $26 \%$ are case studies. Two categories of work are obtained: Political participation of the nursing profession and Virtue and competences; Strategies and challenges to participate in PPs. Similarities are shown between both categories.

Conclusion: Nursing has socio-political activism promoted by nurses in different contexts. To the extent that nursing professionals become aware of political involvement as an expression of socio-political knowledge, their participation favors the public policy process. It is an opportunity for change given their strategic position as referents of care of people in health systems.

Keywords: Nursing; Health Policy; Policy Making.

\section{INTRODUCCIÓN}

Las políticas públicas (PPs) son la expresión concreta y tangible de las acciones de la política, desde Enfermería se consideran como las elecciones de la sociedad por medio de sus gobernantes; y tienen como objetivo, representar asuntos públicos y las formas de otorgamiento de los recursos para alcanzarlo. Para esto, la participación política de personas comunes, profesionales o grupos permite un proceso de mejora continua al proceso de formulación de PPs ${ }^{(1)}$.

La Organización Mundial de la Salud (OMS) mediante la declaración de Adelaida del año 2010 abre la discusión y propone la idea de salud en todas las políticas, lo que realza la labor del sector salud y su importancia en el desarrollo de PPs; también como estrategia para lograr desarrollo social, económico y medioambiental que favorezca la salud y el bienestar de las personas. En ello, los profesionales del sector salud, Enfermería en particular, tiene un rol fundamental, dadas las capacidades adquiridas en su formación profesional, que contribuyen sin duda al proceso de formulación de PPs ${ }^{(2,3)}$.

En este contexto la OMS desde el año 2002 ha publicado periódicamente las "Strategic directions for strengthening nursing and midwifery"(2), donde conforme al contexto histórico explicita la necesidad de participación de los profesionales de Enfermería en la definición de PPs de salud, con el fin de hacerlas más eficientes y eficaces en el logro de los objetivos de salud mundiales. En el informe de Situación de la Enfermería en el mundo $2020^{(4)}$ se menciona que es preciso invertir en educación, empleo y liderazgo en Enfermería, puesto que proporciona una visión y una agenda prospectiva para las políticas; paradójicamente, al tiempo que el mundo celebra en 2020 el Año Internacional de los Profesionales de Enfermería y Partería, se enfrenta la pandemia de enfermedad por coronavirus 2019-20(5).

Los profesionales de Enfermería representan el $56 \%$ de la fuerza laboral de salud total en el mundo, y desarrollan tanto acciones específicas de cuidado de personas como acciones complementarias a las demás áreas de la salud(5); siendo evidente la posesión de competencias diversas para apoyar los procesos de formulación de PPs en Enfermería ${ }^{(1-3,5)}$. Así, apoyadas en el patrón de conocimiento sociopolítico de Enfermería, buscan dar respuesta a problemas que surgen en materia de acceso, calidad de la atención en salud y cobertura sanitaria universal(2,3,5).

El rol de Enfermería ha estado históricamente asociado al cuidado de la salud de las personas y de las comunidades, marcado transversalmente por el activismo político ejercido en forma pionera por Florence Nightingale en el siglo XIX, donde el acto de 
cuidar se basa en valores humanos construidos y la dimensión política, propia de la acción de la profesión, siempre tienen lugar en un contexto históricamente determinado. De este modo, Enfermería tiene un rol fundamental como parte integral del trabajo colectivo(1).

Dentro de los trabajadores de primera línea, Enfermería brinda cuidados abordando desafíos sociosanitarios actuales, como las enfermedades emergentes, una población envejecida, enferma y sin seguro, costos crecientes en planes y seguros, escasez de proveedores y, la desconexión con el proceso político sanitario y las reformas en salud $^{(6)}$. De esta forma, la participación en la formulación de PPs ofrece a Enfermería una oportunidad de influir en programas sociosanitarios diversos, que tienen un profundo impacto en la provisión de salud y prácticas del cuidado de alta calidad en tiempos de grandes encrucijadas sociosanitarias actuales ${ }^{(5)}$.

Es por esto que sería positivo y muy ventajoso más enfermeras fueran desarrolladoras proactivas de PPs, además de implementarlas ${ }^{(6)}$. Al asumir un rol activo, Enfermería tendría liderazgo y protagonismo en el proceso complejo de PPs, fortaleciendo la profesión y la salud colectiva.

Si bien, Enfermería comenzó el camino para lograr una participación en el proceso y formulación de PPs, es prioritario desarrollar un cuerpo de conocimiento que fortalezca el rol de participación política como expresión del patrón de conocimiento socio-político de la profesión, construir formas de pensamiento estratégico a largo plazo para la profesión y motivar a los profesionales de Enfermería a desarrollar el rol político que le corresponde, como estrategia para expandir la participación social y permitir contribuir en el desarrollo de una salud humanizada y una sociedad más sustentable.

En este contexto, se hace necesario el desarrollo de la presente investigación que tiene como objetivo analizar la evidencia científica disponible en la literatura en torno al rol de participación política del profesional enfermero, con el fin de sintetizar la evidencia que sustenta la contribución de la Enfermería al proceso de políticas públicas y construir nuevo conocimiento en esta materia que sea capaz de sustentar futuros procesos investigativos.

\section{METODOLOGÍA}

Este estudio corresponde a una revisión integrativa de la literatura de seis pasos. Las revisiones integrativas permiten resumir la cuantiosa información disponible con respecto al tema tratado, mediante la utilización de diversos tipos de estudios ${ }^{(7)}$.

Siguiendo el camino metodológico: 1) identificación del problema 2) determinación de estrategia de búsqueda y determinación de criterios de inclusión y exclusión 3) definición de la información a ser extraída de los estudios seleccionados /categorización de estudios. 4) evaluación de los estudios incluidos en la revisión integradora 5) interpretación de resultados de los estudios 6) presentación de la revisión/síntesis de conocimiento ${ }^{(7)}$.

La revisión integradora se orientó por medio de la pregunta ¿Cuál debe ser el Rol Político que el profesional enfermero realiza en materia de creación, implementación y 
evaluación de PPs entre los años 2010 y $2020 ?$

Respecto a los criterios de elegibilidad:

- Criterios de inclusión (Cl): Revista científica; texto completo en línea; relación entre Enfermería y política o políticas públicas o formulación de políticas públicas, artículos de análisis políticos - críticos, revisiones de la literatura, estudios de evaluación de procesos estudios cualitativos y estudios de caso.

- Criterios de exclusión (CE): artículos incompletos; artículos en otro idioma que no fuera español, inglés, portugués; no relaciones entre Enfermería con política, artículos con otros profesionales de la salud objetos de estudio.

Para la búsqueda de estudios, se utilizaron la siguiente estrategia de búsqueda: ENFERMERÍA and POLÍTICA DE SALUD and FORMULACIÓN DE POLÍTICAS, en idiomas inglés, portugués y español, entre los años 2010 y 2020. Se usaron las bases de datos: MEDLINE/PUBMED; ISI WEB OF SCIENCE, EBSCOHOST WEB, LILACS y SCIELO. Período de búsqueda: marzo - abril 2020.

Para la selección de los datos se utilizó PRISMA modificado (figura 1) para delimitar los textos finales y realizar la presentación de los datos ${ }^{(8)}$. Paso 1: artículos publicados desde 2010 al 2020. Paso 2: se leyó en detalle el título y el resumen de los artículos, se seleccionaron los artículos de interés según $\mathrm{Cl}$ y $\mathrm{CE}$. Paso 3: se leyeron los textos en extenso agrupando los textos en dos áreas temáticas 1) participación política del profesional enfermero en PPs, características y virtudes; 2) estrategias y Desafíos en materia de participación en PPs. Finalmente se realizó un análisis Temático de Minayo ${ }^{(9)}$ categorizando los hallazgos con una síntesis cualitativa y con una narrativa de los hallazgos. 
Figura 1: Diagrama de flujo PRISMA que resume el proceso de selección de estudios.

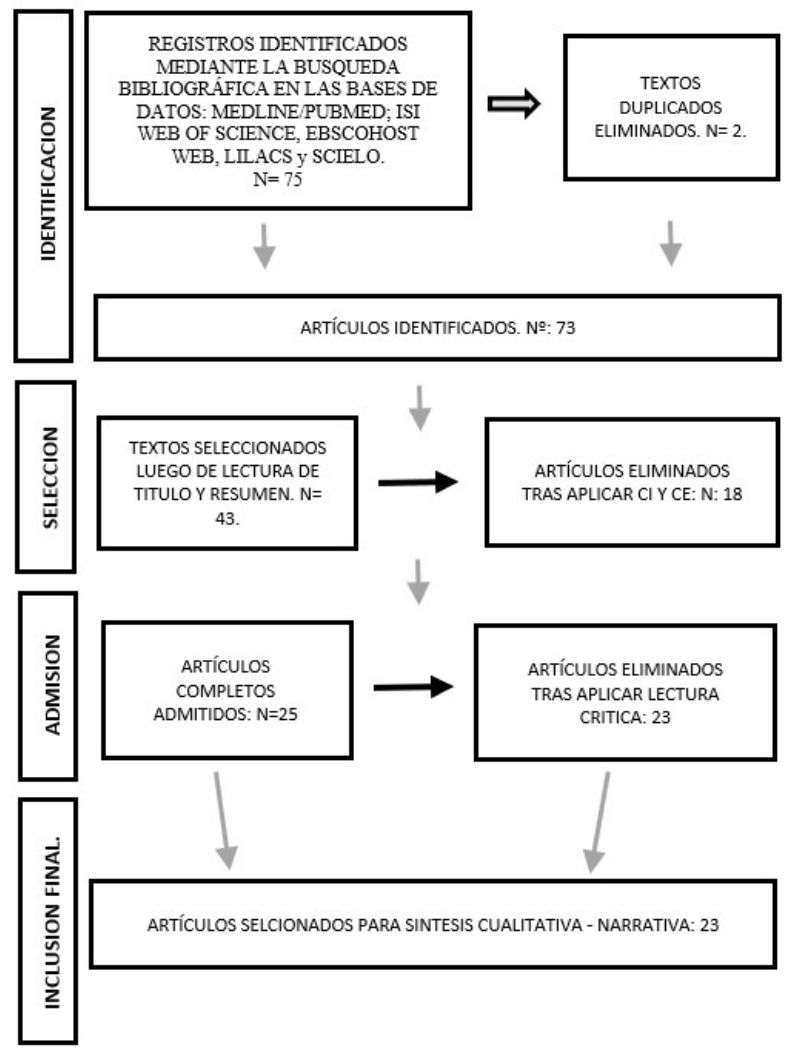

Fuente: Elaboración propia.

\section{RESULTADOS}

\section{Caracterización de los estudios}

De 75 documentos encontrados, 43 cumplieron con criterios de búsqueda. Finalmente, 23 completaron criterios (Figura 1), se presentan en la Tabla 1, 2 y 3.

El análisis descriptivo destaca un $35 \%$ de las publicaciones se realizaron en el período comprendido entre 2010 al 2012; el 48\% fueron llevadas a cabo en Estados Unidos; un $65 \%$ se obtuvieron de PUBMED y el $26 \%$ son estudios de caso. (Tabla 2 ) ambas categorías, destacando en ello el interés de la comunidad investigadora en presentar antecedentes del valor de Enfermería en los diferentes procesos de PPs, al igual que las estrategias necesarias para aprehender estos nuevos conocimientos para enfrentar los nuevos desafíos que subyacen a las dinámicas socio sanitarias actuales. (Tabla 3) 
Tabla 1: Matriz de análisis de artículos seleccionados.

\begin{tabular}{|c|c|c|c|c|c|}
\hline $\mathbf{N}$ & ARTíCULO & $\begin{array}{l}\text { AÑO DE } \\
\text { PUBLICACIÓN } \\
\text { Y PAIS } \\
\end{array}$ & $\begin{array}{l}\text { BASE DE } \\
\text { DATOS }\end{array}$ & $\begin{array}{l}\text { TIPO DE } \\
\text { ESTUDIO }\end{array}$ & OBJETIVO DE ESTUDIO \\
\hline 1 & $\begin{array}{l}\text { The contribution of } \\
\text { nurses to health policy } \\
\text { and advocacy requires } \\
\text { leaders to provide } \\
\text { training and } \\
\text { mentorship. } \\
\text { Authors. Sue Turale } \\
\text { and Wipada } \\
\text { Kunaviktikul } \\
\text { K(10). }\end{array}$ & $\begin{array}{l}\text { 2019, } \\
\text { Tailandia }\end{array}$ & $\begin{array}{l}\text { MEDLI } \\
\text { NE/PUB } \\
\text { MED }\end{array}$ & $\begin{array}{l}\text { Ensayo } \\
\text { reflexivo. }\end{array}$ & $\begin{array}{l}\text { Analizar la contribución de las } \\
\text { enfermeras a las políticas de salud. }\end{array}$ \\
\hline 2 & $\begin{array}{l}\text { Using Ethical } \\
\text { Frameworks in Times } \\
\text { of Transition and } \\
\text { Uncertainty. } \\
\text { Authors. Eileen M. and } \\
\text { Sullivan-Marx }{ }^{(11)} \text {. } \\
\end{array}$ & 2017. USA & $\begin{array}{l}\text { MEDLI } \\
\text { NE/PUB } \\
\text { MED }\end{array}$ & $\begin{array}{l}\text { Ensayo } \\
\text { reflexivo. }\end{array}$ & $\begin{array}{l}\text { Reflexionar en torno al marco ético y } \\
\text { moral utilizado en la dirección de } \\
\text { planificación y la implementación de } \\
\text { PPs y los puntos de vista políticos para } \\
\text { la práctica de enfermería y el liderazgo } \\
\text { organizacional. }\end{array}$ \\
\hline 3 & $\begin{array}{l}\text { Creating academic } \\
\text { structures to promote } \\
\text { nursing's role in global } \\
\text { health policy. } \\
\text { Authors. S Gimbel, P } \\
\text { Kohler, P Mitchell and } \\
\text { A Emami } \\
\end{array}$ & 2017, USA. & $\begin{array}{l}\text { MEDLI } \\
\text { NE/PUB } \\
\text { MED }\end{array}$ & $\begin{array}{l}\text { Revisión } \\
\text { integrati } \\
\text { va de la } \\
\text { literatura }\end{array}$ & $\begin{array}{l}\text { Analizar estructuras académicas como } \\
\text { una forma de ampliar la participación } \\
\text { de la enfermería en el desarrollo de } \\
\text { políticas de salud global. }\end{array}$ \\
\hline 4 & $\begin{array}{l}\text { The Role of the Nurse } \\
\text { Scientist as a } \\
\text { Knowledge Broker. } \\
\text { Authors. Marcella } \\
\text { Remer Thompson and } \\
\text { Donna Schwartz } \\
\text { Barcott }^{(13)} \text {. }\end{array}$ & 2019. USA & $\begin{array}{l}\text { MEDLI } \\
\text { NE/PUB } \\
\text { MED }\end{array}$ & $\begin{array}{l}\text { Revisión } \\
\text { sistemáti } \\
\text { ca de la } \\
\text { literatura } \\
\text {. }\end{array}$ & $\begin{array}{l}\text { Analizar el papel de la enfermera } \\
\text { científica como agente intermediario } \\
\text { del conocimiento (Knowledge Broker). }\end{array}$ \\
\hline 5 & $\begin{array}{l}\text { Why we need multi- } \\
\text { level health workforce } \\
\text { governance: Case } \\
\text { studies from nursing } \\
\text { and medicine in } \\
\text { Germany. } \\
\text { Authors. Ellen } \\
\text { Kuhlmann and Christa } \\
\text { Larsen }^{(14)} \text {. }\end{array}$ & 2015, Suecia. & $\begin{array}{l}\text { MEDLI } \\
\text { NE/PUB } \\
\text { MED }\end{array}$ & $\begin{array}{l}\text { Estudio } \\
\text { de caso }\end{array}$ & $\begin{array}{l}\text { Analizar un enfoque de gobernanza } \\
\text { multinivel e integrador en la gestión } \\
\text { hospitalaria, que tenga como motor } \\
\text { innovador, enfermería y el personal } \\
\text { sanitario. }\end{array}$ \\
\hline 6 & $\begin{array}{l}\text { Evolving trends in } \\
\text { nurse regulation: what } \\
\text { are the policy impacts } \\
\text { for nursing's social } \\
\text { mandate? } \\
\text { Authors. Susan } \\
\text { Duncan, Sally Thorne, } \\
\text { and Patricia Rodney }{ }^{(15)} \text {. }\end{array}$ & 2015, Canadá. & $\begin{array}{l}\text { MEDLI } \\
\text { NE/PUB } \\
\text { MED }\end{array}$ & $\begin{array}{l}\text { Análisis } \\
\text { político } \\
\text { crítico. }\end{array}$ & $\begin{array}{l}\text { Analizar el impacto que el } \\
\text { conocimiento de las implicaciones de } \\
\text { los cambios normativos legislativos y } \\
\text { organizativos generan en la evolución } \\
\text { de la regulación de la enfermería, } \\
\text { como elemento que influye en la } \\
\text { influencia en asuntos urgentes de } \\
\text { salud y políticas públicas sanitarias, } \\
\text { para el logro de la equidad en salud a } \\
\text { nivel mundial. }\end{array}$ \\
\hline
\end{tabular}




\begin{tabular}{|c|c|c|c|c|c|}
\hline 7 & $\begin{array}{l}\text { The contribution of } \\
\text { nursing to high-value } \\
\text { inpatient care } \\
\text { Author. Ellen T } \\
\text { Kurtzman }^{(16)} \text {. }\end{array}$ & 2010, USA & $\begin{array}{l}\text { MEDLI } \\
\text { NE/PUB } \\
\text { MED }\end{array}$ & $\begin{array}{l}\text { Búsqueda } \\
\text { estructu } \\
\text { rada de la } \\
\text { literatura } \\
\text { empírica } \\
\text { revisada } \\
\text { por } \\
\text { pares. }\end{array}$ & $\begin{array}{l}\text { Analizar la contribución de enfermería } \\
\text { a la calidad y el costo de las } \\
\text { atenciones en salud y la participación } \\
\text { política, desde una mirada d la } \\
\text { influencia en políticas públicas y } \\
\text { beneficio social. }\end{array}$ \\
\hline 8 & $\begin{array}{l}\text { Wisdom within: } \\
\text { unlocking the potential } \\
\text { of big data for nursing } \\
\text { regulators. } \\
\text { Authors. L Blumer, C } \\
\text { Giblin, G Lemermeyer } \\
\text { and J A Kwan }{ }^{(17)} \text {. }\end{array}$ & 2017, Canadá & $\begin{array}{l}\text { MEDLI } \\
\text { NE/PUB } \\
\text { MED }\end{array}$ & $\begin{array}{l}\text { Estudio } \\
\text { de caso. }\end{array}$ & $\begin{array}{l}\text { Analizar las implicancias del uso de } \\
\text { macrodatos por parte de enfermería, } \\
\text { como parte fundamental de los } \\
\text { procesos de toma de decisiones y el } \\
\text { desarrollo de política relevante, sólida } \\
\text { y creíble. }\end{array}$ \\
\hline 9 & $\begin{array}{l}\text { Professional advocacy: } \\
\text { linking Virginia's story } \\
\text { to public policy-making } \\
\text { theory, learning from } \\
\text { the past and applying } \\
\text { it to our future. } \\
\text { Author. Melody K } \\
\text { Eaton }^{(18)} \text {. }\end{array}$ & 2012 , USA. & $\begin{array}{l}\text { MEDLI } \\
\text { NE/PUB } \\
\text { MED }\end{array}$ & $\begin{array}{l}\text { Estudio } \\
\text { de caso. }\end{array}$ & $\begin{array}{l}\text { Analizar el vínculo que posee } \\
\text { enfermería con experiencias pasadas, } \\
\text { con el desarrollo de estrategias } \\
\text { específicas para influir de manera } \\
\text { efectiva en los formuladores de } \\
\text { políticas respecto a su valor y el } \\
\text { aporte que pueda entregar a los } \\
\text { cambios en política. }\end{array}$ \\
\hline $\begin{array}{l}1 \\
0\end{array}$ & $\begin{array}{l}\text { Nurses and public } \\
\text { policy. } \\
\text { Author. Debra S } \\
\text { Nault }^{(19)} \text {. }\end{array}$ & 2012 , USA. & $\begin{array}{l}\text { MEDLI } \\
\text { NE/PUB } \\
\text { MED }\end{array}$ & $\begin{array}{l}\text { Estudio } \\
\text { de caso. }\end{array}$ & $\begin{array}{l}\text { Analizar el impacto de las estrategias } \\
\text { de discusión política, en el desarrollo } \\
\text { de políticas relevantes para el } \\
\text { quehacer de enfermería. }\end{array}$ \\
\hline $\begin{array}{l}1 \\
1\end{array}$ & $\begin{array}{l}\text { Postpartum } \\
\text { depression: a chronicle } \\
\text { of health policy } \\
\text { development. } \\
\text { Author. Saralee Glasser } \\
\mathrm{MA}^{(20)} \text {. }\end{array}$ & 2010. Israel. & $\begin{array}{l}\text { MEDLI } \\
\text { NE/PUB } \\
\text { MED }\end{array}$ & $\begin{array}{l}\text { Estudio } \\
\text { de caso. }\end{array}$ & $\begin{array}{l}\text { Describir el impacto que el trabajo de } \\
\text { Enfermería como grupo cohesionado } \\
\text { puede lograr, al utilizar todos los } \\
\text { recursos personales y colectivos en } \\
\text { proyectos que impactan en la } \\
\text { sociedad. }\end{array}$ \\
\hline $\begin{array}{l}1 \\
2\end{array}$ & $\begin{array}{l}\text { Changes in political } \\
\text { astuteness following } \\
\text { nurse legislative day. } \\
\text { Authors. Janet } \\
\text { Primomo and Elin A } \\
\text { Björling } \\
\text { (21). }\end{array}$ & 2013, USA. & $\begin{array}{l}\text { MEDLI } \\
\text { NE/PUB } \\
\text { MED }\end{array}$ & Cohorte & $\begin{array}{l}\text { Analizar el concepto de astucia } \\
\text { política, como un aspecto vital de } \\
\text { la profesión de enfermería que } \\
\text { influye en la participación en el } \\
\text { proceso político. }\end{array}$ \\
\hline $\begin{array}{l}1 \\
3\end{array}$ & $\begin{array}{l}\text { Oregon's Senate Bill } \\
\text { 560: practical policy } \\
\text { lessons for nurse } \\
\text { advocates. } \\
\text { Author. Maria Gilson } \\
\text { Sistrom } \\
\text { (22). }\end{array}$ & 2010, USA. & $\begin{array}{l}\text { MEDLI } \\
\text { NE/PUB } \\
\text { MED }\end{array}$ & $\begin{array}{l}\text { Análisis } \\
\text { de } \\
\text { conteni } \\
\text { do } \\
\text { cualitati } \\
\text { vo de } \\
\text { documen } \\
\text { tos y } \\
\text { entrevis } \\
\text { tas } \\
\text { semies } \\
\text { tructura } \\
\text { das. }\end{array}$ & $\begin{array}{l}\text { Analizar el concepto nurse lobbyist, } \\
\text { como elemento crucial para un } \\
\text { cambio exitoso en las políticas de } \\
\text { salud. }\end{array}$ \\
\hline
\end{tabular}




\begin{tabular}{|c|c|c|c|c|c|}
\hline $\begin{array}{l}1 \\
4\end{array}$ & $\begin{array}{l}\text { Moving policies } \\
\text { upstream to mitigate } \\
\text { the social } \\
\text { determinants of early } \\
\text { childbearing } \\
\text { Author. Lee Smith } \\
\text { Battle }{ }^{(23)} \text {. }\end{array}$ & 2012 , USA & $\begin{array}{l}\text { MEDLI } \\
\text { NE/PUB } \\
\text { MED }\end{array}$ & $\begin{array}{l}\text { Análisis } \\
\text { de } \\
\text { conteni } \\
\text { do } \\
\text { cualitati } \\
\text { vo de } \\
\text { documen } \\
\text { tos. }\end{array}$ & $\begin{array}{l}\text { Determinar cómo las políticas públicas } \\
\text { ascendentes y lideradas por } \\
\text { enfermería, pueden reducir las } \\
\text { amplias y crecientes desigualdades en } \\
\text { educación, ingresos y salud entre los } \\
\text { ciudadanos. }\end{array}$ \\
\hline $\begin{array}{l}1 \\
5\end{array}$ & $\begin{array}{l}\text { Development of } \\
\text { community nursing in } \\
\text { Zhejiang Province, } \\
\text { China: a report of the } \\
\text { driving measures. } \\
\text { Authors. W Fu, J Bao } \\
\text { and J Meng }{ }^{(24)} \text {. }\end{array}$ & 2010, China. & $\begin{array}{l}\text { MEDLI } \\
\text { NE/PUB } \\
\text { MED }\end{array}$ & $\begin{array}{l}\text { Estudio } \\
\text { de caso. }\end{array}$ & $\begin{array}{l}\text { Describir el trabajo preveniente de la } \\
\text { enfermería comunitaria con enfoque } \\
\text { múltiple, como estrategia para } \\
\text { intensionar cambios importantes en } \\
\text { materia satisfacer las necesidades } \\
\text { cambiantes de la sociedad, desde los } \\
\text { territorios. }\end{array}$ \\
\hline $\begin{array}{l}1 \\
6\end{array}$ & $\begin{array}{l}\text { Promoting Population } \\
\text { Health Nurse } \\
\text { Advocacy, Policy } \\
\text { Making, and Use of } \\
\text { Media. } \\
\text { Autor: Myers, Carole } \\
\mathrm{R}^{(1)} \text {. }\end{array}$ & 2020, USA. & $\begin{array}{l}\text { ISI WEB } \\
\text { OF } \\
\text { SCIENCE }\end{array}$ & $\begin{array}{l}\text { Análisis } \\
\text { de } \\
\text { conteni } \\
\text { do } \\
\text { cualitati } \\
\text { vo de } \\
\text { documen } \\
\text { tos }\end{array}$ & $\begin{array}{l}\text { Describir la importancia de avanzar en } \\
\text { una intensa definición de los roles de } \\
\text { enfermería relacionados con la } \\
\text { promoción y su participación en los } \\
\text { procesos de formulación de políticas } \\
\text { públicas, para avanzar en la salud de la } \\
\text { población, a través de los medios de } \\
\text { comunicación, como estrategia para } \\
\text { difundir definiciones de problemas de } \\
\text { individuos, familias y comunidades. }\end{array}$ \\
\hline $\begin{array}{l}1 \\
7\end{array}$ & $\begin{array}{l}\text { Using narratives to } \\
\text { impact health policy- } \\
\text { making: a systematic } \\
\text { review } \\
\text { Autor(es): Fadlallah, } \\
\text { Racha; El-Jardali, Fadi; } \\
\text { Nomier, Mohamed; } \\
\text { Hemadi, Nour; Arif, } \\
\text { Khurram; Langlois, } \\
\text { Etienne V and Akl, Elie } \\
A^{(25)} \text {. }\end{array}$ & 2019, Líbano. & $\begin{array}{l}\text { ISI WEB } \\
\text { OF } \\
\text { SCIENCE }\end{array}$ & $\begin{array}{l}\text { Revisión } \\
\text { sistemáti } \\
\text { ca de la } \\
\text { literatura }\end{array}$ & $\begin{array}{l}\text { Analizar los usos de intervenciones } \\
\text { narrativas, como estrategia } \\
\text { comunicacional de enfermería con } \\
\text { influencia positiva en el proceso de } \\
\text { formulación de políticas de salud. }\end{array}$ \\
\hline $\begin{array}{l}1 \\
8\end{array}$ & $\begin{array}{l}\text { Nurse Knowledge and } \\
\text { Engagement in Health } \\
\text { Policy Making: Findings } \\
\text { From a Pilot Study } \\
\text { Authors: Lewinski, } \\
\text { Allison and Simmons, } \\
\text { Leigh Ann }{ }^{(26)} \text {. }\end{array}$ & 2018. USA. & $\begin{array}{l}\text { ISI WEB } \\
\text { OF } \\
\text { SCIENCE }\end{array}$ & $\begin{array}{l}\text { Estudio } \\
\text { descripti } \\
\text { vo de } \\
\text { corte } \\
\text { transver } \\
\text { sal con } \\
\text { un plan } \\
\text { de } \\
\text { muestreo } \\
\text { por } \\
\text { conve } \\
\text { niencia } \\
\text { para } \\
\text { encues } \\
\text { tar a } \\
\text { enferme }\end{array}$ & $\begin{array}{l}\text { Describir la importancia de apoyar a } \\
\text { las enfermeras en el conocimiento en } \\
\text { materia de dinámicas políticas } \\
\text { actuales o sobre la promoción de } \\
\text { políticas de salud, para influir en la } \\
\text { defensa de las políticas de salud y } \\
\text { mejorar los resultados de salud de los } \\
\text { pacientes y las comunidades. }\end{array}$ \\
\hline
\end{tabular}




\begin{tabular}{|c|c|c|c|c|c|}
\hline & & & & $\begin{array}{l}\text { ros en } \\
\text { ejercicio. }\end{array}$ & \\
\hline $\begin{array}{l}1 \\
9\end{array}$ & $\begin{array}{l}\text { Exploration of priority } \\
\text { actions for } \\
\text { strengthening the role } \\
\text { of nurses in achieving } \\
\text { universal health } \\
\text { coverage. } \\
\text { Authors: Maaitah, } \\
\text { Rowaida Al and } \\
\text { AbuAlRub, Raeda } \\
\text { Fawzi } 27 \text {. }\end{array}$ & $\begin{array}{l}\text { 2017, } \\
\text { Jordania. }\end{array}$ & LILACS & $\begin{array}{l}\text { Diseño } \\
\text { cualitati } \\
\text { vo } \\
\text { explora } \\
\text { torio, } \\
\text { utilizan } \\
\text { do una } \\
\text { encues } \\
\text { ta semi- } \\
\text { estructu } \\
\text { rada }\end{array}$ & $\begin{array}{l}\text { Exploración de acciones } \\
\text { prioritarias para fortalecer el rol de } \\
\text { las enfermeras en el logro de la } \\
\text { cobertura universal de salud. }\end{array}$ \\
\hline $\begin{array}{l}2 \\
0\end{array}$ & $\begin{array}{l}\text { Pattern of socio- } \\
\text { political knowledge in } \\
\text { nursing: conceptual } \\
\text { reflections. } \\
\text { Authors: Osorio } \\
\text { Castaño, Jhon Henry }{ }^{(3)} \text {. }\end{array}$ & $\begin{array}{l}\text { 2016, } \\
\text { Colombia. }\end{array}$ & LILACS & $\begin{array}{l}\text { Análisis } \\
\text { de } \\
\text { conteni } \\
\text { do } \\
\text { cualitati } \\
\text { vo de } \\
\text { documen } \\
\text { tos. }\end{array}$ & $\begin{array}{l}\text { Analizar el patrón de conocimiento } \\
\text { sociopolítico con potenciador de la } \\
\text { práctica y la investigación de } \\
\text { enfermería. }\end{array}$ \\
\hline $\begin{array}{l}2 \\
1\end{array}$ & 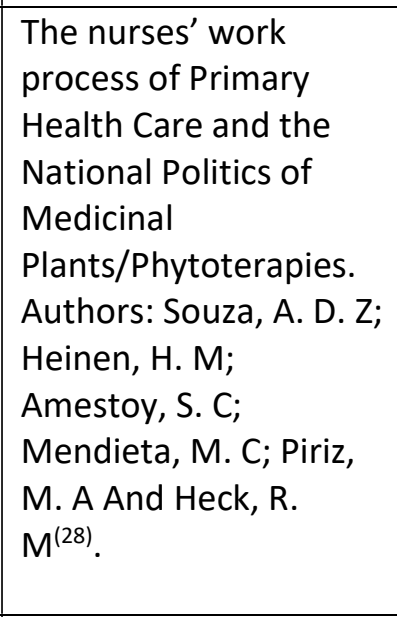 & 2016, Brasil. & LILACS & $\begin{array}{l}\text { Estudio } \\
\text { cualitati } \\
\text { vo, } \\
\text { descripti } \\
\text { vo. Datos } \\
\text { recolecta } \\
\text { dos a } \\
\text { través de } \\
\text { un } \\
\text { cuestiona } \\
\text { rio } \\
\text { autoadmi } \\
\text { nistrado }\end{array}$ & $\begin{array}{l}\text { Analizar el intercambio de } \\
\text { experiencias entre profesionales de } \\
\text { enfermería y la comunidad, como } \\
\text { estrategia para expandir y fortalecer } \\
\text { las relaciones interprofesionales que } \\
\text { enriquece el trabajo en la comunidad. }\end{array}$ \\
\hline $\begin{array}{l}2 \\
2\end{array}$ & $\begin{array}{l}\text { Permanen health } \\
\text { education: } \\
\text { constructions of nurses } \\
\text { rom family health } \\
\text { strategy. } \\
\text { Authors: Ribeiro dos } \\
\text { Santos, Adilson and } \\
\text { Lemos Coutinho, } \\
\text { Márcio }\end{array}$ & 2014, Brasil & LILACS & $\begin{array}{l}\text { Estudio } \\
\text { descripti } \\
\text { vo, } \\
\text { explorato } \\
\text { rio, con } \\
\text { abordaje } \\
\text { cualitati } \\
\text { vo. }\end{array}$ & $\begin{array}{l}\text { Analizar la educación permanente en } \\
\text { salud, como factor esencial para el } \\
\text { desarrollo de los trabajadores } \\
\text { sanitaros en general y de enfermería } \\
\text { en particular. }\end{array}$ \\
\hline $\begin{array}{l}2 \\
3\end{array}$ & $\begin{array}{l}\text { Brazilian nursing and } \\
\text { the democratization of } \\
\text { health: notes on the } \\
\text { National Policy of } \\
\text { Popular Education in } \\
\text { Health. } \\
\text { Authors: David, Helena } \\
\text { Maria Scherlowski } \\
\text { Leal; Bonetti, Osvaldo } \\
\text { Peralta; }\end{array}$ & 2012, Brasil. & LILACS & $\begin{array}{l}\text { Ensayo } \\
\text { reflexivo. }\end{array}$ & $\begin{array}{l}\text { Analizar el debate y el diálogo para } \\
\text { intensionar cambios sociales más } \\
\text { profundos, liderados por enfermería. }\end{array}$ \\
\hline
\end{tabular}




\begin{tabular}{|l|l|l|l|l|l|}
\hline $\begin{array}{l}\text { and Silva, Maria } \\
\text { Rocineide Ferreira } \\
\mathrm{da}^{(30)} .\end{array}$ & 0 & $\begin{array}{l}\text { EBSCOH } \\
\text { OST } \\
\text { WEB }\end{array}$ & 0 & 0 \\
\hline $\mathbf{0}$ & 0 & 0 & SCIELO. & 0 & 0 \\
\hline $\mathbf{0}$ & 0 & 0 & & & \\
\hline
\end{tabular}

Fuente: Elaboración propia.

Tabla 2. Síntesis cualitativa - descriptiva de los textos.

\begin{tabular}{|c|c|c|}
\hline Características & Categorías & Cantidad. \\
\hline \multirow[t]{4}{*}{ Año de publicación. } & $2010-2012$ & 8 \\
\hline & $2013-2015$ & 4 \\
\hline & $2016-2018$ & 7 \\
\hline & $2019-2020$ & 4 \\
\hline \multirow[t]{10}{*}{ Diseño de estudio } & Estudio de caso & 6 \\
\hline & Análisis cualitativo de documentos. & 4 \\
\hline & Estudio cualitativo. & 3 \\
\hline & Ensayo reflexivo & 3 \\
\hline & Revisión sistemática & 2 \\
\hline & Revisión integrativa de la literatura. & 1 \\
\hline & Análisis crítico & 1 \\
\hline & Búsqueda estructurada de la literatura & 1 \\
\hline & Cohorte & 1 \\
\hline & Cross-seccional & 1 \\
\hline \multirow[t]{6}{*}{ País de origen } & Estados Unidos. & 11 \\
\hline & Brasil. & 3 \\
\hline & Canadá. & 2 \\
\hline & China & 1 \\
\hline & Colombia & 1 \\
\hline & Suecia, Tailandia, Líbano, Israel y Jordania. & 5 \\
\hline \multirow[t]{5}{*}{ Base de datos } & MEDLINE/PUBMED & 15 \\
\hline & ISI WEB OF SCIENCE & 3 \\
\hline & EBSCOHOST WEB & 0 \\
\hline & LILACS & 5 \\
\hline & SCIELO & 0 \\
\hline
\end{tabular}

Fuente: Elaboración propia. 
Tabla 3: Ejes temáticos surgidos del análisis.

\begin{tabular}{|c|c|}
\hline Categorías & Artículos asociados. \\
\hline \multirow{15}{*}{$\begin{array}{l}\text { 1. Participación } \\
\text { política del } \\
\text { profesional } \\
\text { enfermero. } \\
\text { Virtudes y } \\
\text { competencias. }\end{array}$} & $\begin{array}{l}\text { 1. Creating academic structures to promote nursing's role in global } \\
\text { health policy. }\end{array}$ \\
\hline & 2. The Role of the Nurse Scientist as a Knowledge Broker. \\
\hline & $\begin{array}{l}\text { 3. Evolving trends in nurse regulation: what are the policy impacts for } \\
\text { nursing's social mandate? }\end{array}$ \\
\hline & 4. The contribution of nursing to high-value inpatient care. \\
\hline & $\begin{array}{l}\text { 5. Wisdom within: unlocking the potential of big data for nursing } \\
\text { regulators. }\end{array}$ \\
\hline & 6. Nurses and public policy. \\
\hline & 7. Postpartum depression: a chronicle of health policy development. \\
\hline & 8. Changes in political astuteness following nurse legislative day. \\
\hline & $\begin{array}{l}\text { 9. Development of community nursing in Zhejiang Province, China: a } \\
\text { report of the driving measures. }\end{array}$ \\
\hline & $\begin{array}{l}\text { 10. Promoting Population Health Nurse Advocacy, Policy Making, and } \\
\text { Use of Media. }\end{array}$ \\
\hline & $\begin{array}{l}\text { 11. Nurse Knowledge and Engagement in Health Policy Making: Findings } \\
\text { From a Pilot Study. }\end{array}$ \\
\hline & $\begin{array}{l}\text { 12. Exploration of priority actions for strengthening the role of nurses in } \\
\text { achieving universal health coverage. }\end{array}$ \\
\hline & $\begin{array}{l}\text { 13. Pattern of socio-political knowledge in nursing: conceptual } \\
\text { reflections. }\end{array}$ \\
\hline & $\begin{array}{l}\text { 14. The nurses' work process of Primary Health Care and the National } \\
\text { Politics of Medicinal Plants/Phytoterapies. }\end{array}$ \\
\hline & $\begin{array}{l}\text { 15. Brazilian nursing and the democratization of health: notes on the } \\
\text { National Policy of Popular Education in Health }\end{array}$ \\
\hline \multirow{13}{*}{$\begin{array}{l}\text { 2. Estrategias y } \\
\text { desafíos para } \\
\text { participar en } \\
\text { PPs. }\end{array}$} & $\begin{array}{l}\text { 1. Creating academic structures to promote nursing's role in global } \\
\text { health policy. }\end{array}$ \\
\hline & $\begin{array}{l}\text { 2. he contribution of nurses to health policy and advocacy requires } \\
\text { leaders to provide training and mentorship. }\end{array}$ \\
\hline & 3. Using Ethical Frameworks in Times of Transition and Uncertainty. \\
\hline & $\begin{array}{l}\text { 4. Why we need multi-level health workforce governance: Case studies } \\
\text { from nursing and medicine in Germany. }\end{array}$ \\
\hline & $\begin{array}{l}\text { 5. Evolving trends in nurse regulation: what are the policy impacts for } \\
\text { nursing's social mandate? Authors: Susan Duncan, Sally Thorne and } \\
\text { Patricia Rodney. }\end{array}$ \\
\hline & $\begin{array}{l}\text { 6. Wisdom within: unlocking the potential of big data for nursing } \\
\text { regulators. }\end{array}$ \\
\hline & $\begin{array}{l}\text { 7. Professional advocacy: linking Virginia's story to public policy-making } \\
\text { theory, learning from the past and applying it to our future. }\end{array}$ \\
\hline & 8. Changes in political astuteness following nurse legislative day. \\
\hline & 9. Oregon's Senate Bill 560: practical policy lessons for nurse advocates. \\
\hline & $\begin{array}{l}\text { 10. Moving policies upstream to mitigate the social determinants of early } \\
\text { childbearing. }\end{array}$ \\
\hline & $\begin{array}{l}\text { 11. Promoting Population Health Nurse Advocacy, Policy Making, and } \\
\text { Use of Media. }\end{array}$ \\
\hline & 12. Using narratives to impact health policy-making: a systematic review. \\
\hline & $\begin{array}{l}\text { 13. Nurse Knowledge and Engagement in Health Policy Making: Findings } \\
\text { From a Pilot Study. }\end{array}$ \\
\hline
\end{tabular}




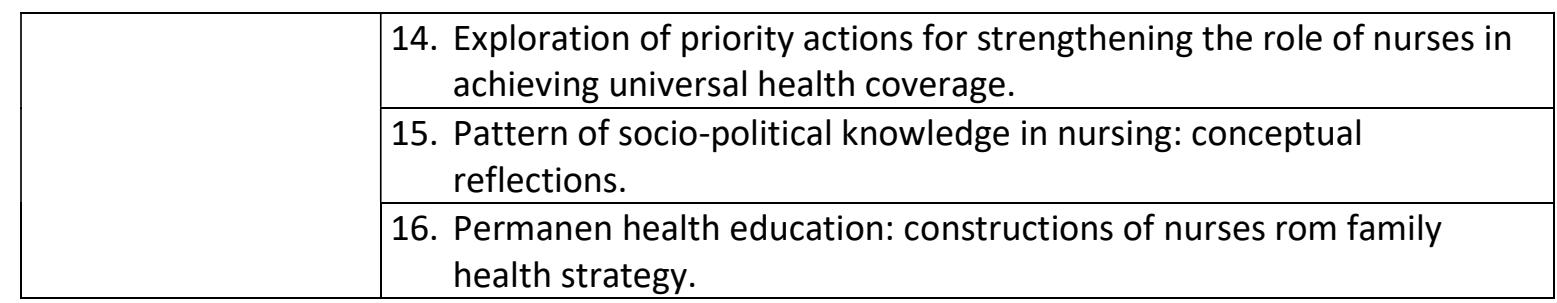

Fuente: Elaboración propia.

\section{DISCUSIÓN}

Al analizar la producción científica sobre participación política y Enfermería, se destaca el interés de la comunidad investigadora en presentar antecedentes del valor de Enfermería en los diferentes procesos de PPs. De igual manera, se destacan también las estrategias necesarias para aprehender conocimientos que permitan enfrentar los nuevos desafíos que subyacen a las dinámicas socio sanitarias actuales. De esta manera, la conquista de espacios de participación política y la consolidación del patrón de conocimiento socio político a partir de un continuo proceso de aprendizaje y la práctica profesional, favorecerán el cambio y mejoras sustantivas de las dinámicas socio sanitarias actuales ${ }^{(3)}$.

\section{Virtudes y competencias profesionales para potenciar la participación política del profesional enfermero}

La Enfermería, debido a su importancia histórica, social, su inserción generalizada en los espacios para la producción de atención individual y colectiva y el patrón de conocimiento sociopolítico que le es propio; constituye una práctica social que puede avanzar para componer fuerzas capaces de movilizar cambios sociales más amplios $^{(3)}$.

Esta profesión se caracteriza por un alto beneficio social, así como su elevada contribución a la calidad y el costo de las atenciones en salud; perfilándose como segura, eficaz, centrada en el paciente, oportuna, eficiente y equitativa ${ }^{(16,19)}$. Las enfermeras quienes poseen un alto estándar formativo, pueden "leer el mundo"(23) y tienen experiencia especializada en temas importantes de amplio interés. El público confía en las enfermeras y las reconoce como los principales actores de la atención sanitaria(1).

Como profesión disciplinar basada en el cuidado, las enfermeras están en una posición única para abogar por los pacientes y sus familias ${ }^{(19)}$, identificar problemas de salud públicas ${ }^{(20)}$ y encontrar soluciones desde el trabajo que realizan ${ }^{(19)}$.

De este modo, pueden desarrollar intensamente la astucia política como un aspecto vital de la profesión de Enfermería relacionada con el rol de participación política, el cual incluye: comprensión del proceso legislativo y de políticas públicas, conocimiento y habilidades políticas necesarias para abogar por las personas y las comunidades, saber quiénes son los responsables de la formulación de políticas y cómo comunicarse con ellos. Ante lo cual, la conciencia de este rol es el primer paso para mejorar la capacidad de las enfermeras para apoyar las políticas gubernamentales y tener una participación activa en el proceso político(21). 
Involucrar a la Enfermería en el desarrollo de políticas de salud global es vital para asegurar la ampliación de programas de salud efectivos y una salud global sólida(12). Es por esto que, participar en materia política dada su comprensión de las implicaciones de los cambios normativos, legislativos y organizativos en el entorno sanitario $^{(19)}$ y de los territorios ${ }^{(24)}$ requeriría de tres estrategias para influir aún más en el proceso político: desarrollar un conocimiento profundo en los subsistemas políticos; la construcción de redes basadas en un espíritu democrático y la participación en el área de políticas durante períodos de tiempo prolongados ${ }^{(15)}$.

Las enfermeras pueden participar a lo largo de todo el ciclo de formulación de políticas, promoviendo aquellas basadas, tanto en la evidencia científica obtenida desde el uso de macrodatos ${ }^{(17)}$, como la empírica obtenida desde la experiencia

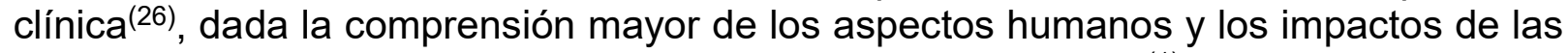
políticas propuestas y existentes en todos los niveles sanitarios ${ }^{(1)}$.

En este sentido, se necesita trabajar de modo expansivo en un enfoque más allá de las aspiraciones profesionales y abogar más por la relevancia política de su quehacer, que los beneficios individuales(19). En ello, los autores destacan el ejercicio de diálogo ${ }^{(16,19)}$ y que la visibilización de su quehacer ${ }^{(27)}$, a través del uso de redes sociales y las comunicaciones ${ }^{(1)}$, podría mejorar la capacidad de las enfermeras para abogar en el ámbito político-legislativo por sus poblaciones de interés y, a su vez, lograr el objetivo de mejorar los resultados de salud de las comunidades ${ }^{(26)}$.

\section{Estrategias y desafíos para participar en política y el desarrollo de PPs}

Los autores posicionan a la Enfermería como una de las profesiones claves en el cumplimiento de las metas de Salud Global; jugando así un rol principal en el logro de acceso universal en salud y el desarrollo sostenible de las comunidades ${ }^{(26,27)}$.

Para poder ejercer una influencia de manera eficaz en materia de PPs, los autores refuerzan la idea de que Enfermería aporta es un aporte en beneficio de la sociedad al contemplar el contexto como una oportunidad de visibilización y expresión, de cambio y transformación ${ }^{(3)}$. A partir del patrón de conocimiento sociopolítico, Enfermería encontrará el camino para avanzar y garantizar el fortalecimiento de la profesión ${ }^{(3)}$, sin embargo, requiere de un consolidado marco regulatorio que respalde sus funciones profesionales y sindicales de manera efectivamente ${ }^{(15) ;}$ también la exposición a los conceptos de formulación de políticas mediante la educación continua(12,15,18,21,26,27,29); la toma de conciencia sobre cuestiones políticas y el desarrollo de astucia política; un sentido de autoeficacia con respecto a la participación en el proceso político ${ }^{(12,15,29)}$; el desarrollo de habilidades de liderazgo y toma de decisiones ${ }^{(11,14,27)}$; comunicación, colaboración y consensos ${ }^{(59)}$ que desafíen los supuestos ideológicos que impulsan las políticas superiores y aboguen por reformas integrales que reduzcan las amplias y crecientes desigualdades en educación, ingresos y salud entre los ciudadanos y las comunidades ${ }^{(23)}$.

En tiempos de grandes cambios en la dirección de PPs, en los que Enfermería se enfrenta a diversos puntos de vista políticos, es preciso que utilice como guía fundamental, el conocimiento empírico en combinación con el juicio ético y moral para apoyar el proceso de planificación e implementación de políticas públicas ${ }^{(23)}$. En este sentido, es necesario según los autores fomentar la realización de acciones coordinadas o coaliciones entre congregaciones y agrupaciones de Enfermería y el 
trabajo interprofesional(12), establecer un régimen interdisciplinario con instituciones, gremios y/o políticos que compartan las mismas preocupaciones y posean objetivos cercanos para el desarrollo de soluciones uniformes y unificadas frente a ventanas de oportunidad $^{(17,18)}$, y, al mismo tiempo, trabajar por la construcción de evidencia científica que permita fortalecer el quehacer profesional(3). Otros autores enfatizan también en el uso de las tecnologías y los medios de comunicación masiva, para dar testimonio, comunicar activamente la perspectiva de Enfermería y participar del dialogo social, frente al acontecer público ${ }^{(1)}$.

Asimismo, es de importancia la formación profesional en políticas públicas, para aportar al trabajo colectivo(1). Otros estudios exponen la formación de enfermeras lobistas $^{(22)}$, comunicadoras ${ }^{(1)}$, intermediarias del conocimiento que conecte la ciencia y la sociedad mediante la construcción de redes, facilitando oportunidades entre los productores y usuarios del conocimiento(27); y también como narradoras para apoyar en el proceso de formulación de políticas públicas, teniendo en consideración diversos enfoques eficaces e innovadores para cerrar la brecha entre la investigación y la formulación de política ${ }^{(25)}$.

No obstante, su impacto en la sociedad, aún persisten desafíos en materia de las barreras para el desarrollo del liderazgo y participación en los procesos políticos ${ }^{(27)}$. En ello, se destaca la falta de apoyo (muros burocráticos), recursos y tiempo para que las enfermeras desarrollen el rol en sus lugares de trabajo. También se devela cierta pasividad, falta de coaliciones y desconocimiento en materia política ${ }^{(29)}$.

Por lo tanto, en la medida en que los profesionales de Enfermería se hacen conscientes del conocimiento del contexto y de las oportunidades que brinda la política como mediador de cambios, este patrón de conocimiento se convertirá en un potenciador de la práctica y la investigación enfermera ${ }^{(3)}$.

\section{CONSIDERACIONES FINALES}

En "el año de la Enfermería", se hace prioritario destacar el rol llevado a cabo por estos profesionales hacia el logro de la salud, el bienestar y el logro de los objetivos de desarrollo sostenible. El desarrollo de la presente revisión permitió analizar la evidencia científica disponible en la literatura en torno al rol de participación política del profesional enfermero, sintetizado la evidencia que sustenta la contribución de la Enfermería el proceso de políticas públicas para construir nuevo conocimiento en esta materia y que, a la vez, sustente futuros procesos investigativos.

En este contexto, se entiende que Enfermería como defensores de la calidad, la seguridad y el acceso a la atención, tienen un vínculo directo con la defensa de las personas, sus familias y comunidades con un sentido ético profesional único; no obstante, para que tomen su lugar en la mesa de toma de decisiones y se involucren profundamente en la formulación de políticas gubernamentales y las reformas en salud, deben desarrollar mayores conocimientos, habilidades y actitudes necesarios en materia de políticas públicas. En este sentido, la gran carga histórica que Enfermería posee, su amplia representación en la fuerza laboral en salud, la alta estima social que cuenta y su amplio conocimiento de las dinámicas socio sanitarias actuales, le otorgan grandes capacidades para apoyar y proporcionar una mayor 
solidez y consistencia al desempeño de las políticas públicas sanitarias y responder de manera más eficiente a las futuras necesidades de salud de la población.

Enfermería constituye una práctica social que puede avanzar para componer fuerzas capaces de movilizar cambios sociales más amplios. Sin duda, en la medida que Enfermería marque su rumbo profesional aprovechando estas ventanas de oportunidad, obtendrá una influencia constructiva en la salud humana y la equidad en salud a nivel mundial.

\section{REFERENCIAS}

1. Myers CR. Promoting Population Health: Nurse Advocacy, Policy Making, and Use of Media. Nurs Clin. 2020;55(1):11-20.

2. Organization $\mathrm{WH}$. Global strategic directions for strengthening nursing and midwifery 2016-2020. 2016 [citado 25 de septiembre de 2020]; Disponible en: https://www.who.int/hrh/nursing_midwifery/global-strategic-midwifery2016-2020.pdf

3. Osorio Castaño JH. Patrón de conocimiento socio-político en enfermería: reflexiones conceptuales. Rev Cuid. 2016;7(2):1352-7.

4. World Health Organization. State of the world's nursing 2020: investing in education, jobs and leadership. 2020 [citado 25 de septiembre de 2020]; Disponible en: https://apps.who.int/iris/bitstream/handle/10665/331677/9789240003279-eng.pdf

5. Cassiani SHDB, Jimenez EFM, Ferreira AU, Peduzzi M, Hernández CL. La situación de la enfermería en el mundo y la Región de las Américas en tiempos de la pandemia de COVID-19. Rev Panam Salud Pública [Internet]. 2020 [citado 17 de mayo de 2020];44. Disponible en: https://www.ncbi.nlm.nih.gov/pmc/articles/PMC7213064/

6. Hassmiller SB. The future of nursing: Lessons in crafting successful policies. 2013 [citado 25 de septiembre de 2020];14(2):55-6. Disponible en: http://citeseerx.ist.psu.edu/viewdoc/download?doi=10.1.1.1034.6705\&rep=rep1\&type= pdf

7. Souza MT de, Silva MD da, Carvalho R de. Revisão integrativa: o que é e como fazer. Einstein São Paulo [Internet]. 2010 [citado 25 de septiembre de 2020];8(1):1026. Disponible en: https://www.scielo.br/pdf/eins/v8n1/pt_1679-4508-eins-8-1-0102

8. Hutton B, Catala-Lopez F, Moher D. The PRISMA statement extension for systematic reviews incorporating network meta-analysis: PRISMA-NMA. Med Clin Barc [Internet]. 2016 [citado 25 de septiembre de 2020];147(6):262-6. Disponible en: file:///C:/Users/denis/AppData/Local/Temp/the-prisma-statement-extension-forsystematic-reviews-incorporating-network-meta-analysis-prisma-nma.pdf

9. De Souza Minayo MC. O desafio do conhecimento. Pesquisa qualitativa em saúde. $13^{\circ}$ edição. Hucitec: São Paulo; 2014.

10. Turale S, Kunaviktikul W. The contribution of nurses to health policy and advocacy requires leaders to provide training and mentorship. Int Nurs Rev [Internet]. 2019 [citado 4 de octubre de 2020];66(3):302-4. Disponible en: https://onlinelibrary.wiley.com/doi/pdf/10.1111/inr.12550

11. Sullivan-Marx EM. Using ethical frameworks in times of transition and uncertainty. J Gerontol Nurs [Internet]. 2017 [citado 27 de septiembre de 2020];43(3):8-12. Disponible en: https://www.researchgate.net/profile/Eileen_SullivanMarx/publication/314143849_Using_Ethical_Frameworks_in_Times_of_Transition_an d_Uncertainty/links/59dcfc974585158eaa08a432/Using-Ethical-Frameworks-in-Timesof-Transition-and-Uncertainty.pdf 
12. Gimbel $S$, Kohler $P$, Mitchell $P$, Emami A. Creating academic structures to promote nursing's role in global health policy. Int Nurs Rev [Internet]. 2017 [citado 27 de septiembre de 2020];64(1):117-25. Disponible en: https://onlinelibrary.wiley.com/doi/pdf/10.1111/inr.12338

13. Thompson MR, Schwartz Barcott $D$. The role of the nurse scientist as a knowledge broker. J Nurs Scholarsh [Internet]. 2019 [citado 27 de septiembre de 2020];51(1):26-39. Disponible https://sigmapubs.onlinelibrary.wiley.com/doi/abs/10.1111/jnu.12439

14. Kuhlmann E, Larsen C. Why we need multi-level health workforce governance: Case studies from nursing and medicine in Germany. Health Policy. 2015;119(12):1636-44.

15. Duncan S, Thorne S, Rodney P. Evolving trends in nurse regulation: what are the policy impacts for nursing's social mandate? Nurs Inq. 2015;22(1):27-38.

16. Kurtzman ET. The contribution of nursing to high-value inpatient care. Policy Polit Nurs Pract. 2010;11(1):36-61.

17. Blumer L, Giblin C, Lemermeyer G, Kwan JA. Wisdom within: Unlocking the potential of big data for nursing regulators. Int Nurs Rev. 2017;64(1):77-82.

18. Eaton MK. Professional advocacy: Linking Virginia's story to public policymaking theory, learning from the past and applying it to our future. Policy Polit Nurs Pract. 2012;13(2):105-12.

19. Nault DS. Nurses and public policy. Mich Nurse. 2012;85(1):13-20.

20. Glasser S. Postpartum depression: a chronicle of health policy development. Isr J Psychiatry Relat Sci [Internet]. 2010 [citado 27 de septiembre de 2020];47(4):254. Disponible

en: https://www.researchgate.net/profile/Saralee_Glasser/publication/49788504_Postpartu m_Depression_A_Chronicle_of_Health_Policy_Development/links/0c96051d111d308bf 3d000000/Postpartum-Depression-A-Chronicle-of-Health-Policy-Development.pdf

21. Primomo J, Björling EA. Changes in political astuteness following nurse legislative day. Policy Polit Nurs Pract. 2013;14(2):97-108.

22. Gilson Sistrom M. Oregon's Senate Bill 560: practical policy lessons for nurse advocates. Policy Polit Nurs Pract. 2010;11(1):29-35.

23. SmithBattle L. Moving policies upstream to mitigate the social determinants of early childbearing. Public Health Nurs. 2012;29(5):444-54.

24. Fu W, Bao J, Meng J. Development of community nursing in Zhejiang Province, China: a report of the driving measures. Int Nurs Rev [Internet]. 2010 [citado 27 de septiembre de 2020];57(2):265-8. Disponible en: https://onlinelibrary.wiley.com/doi/pdf/10.1111/j.1466-7657.2009.00787.x

25. Fadlallah R, El-Jardali F, Nomier M, Hemadi N, Arif K, Langlois EV, et al. Using narratives to impact health policy-making: a systematic review. Health Res Policy Syst [Internet]. 2019 [citado 27 de septiembre de 2020];17(1):26. Disponible en: https://link.springer.com/article/10.1186/s12961-019-0423-4

26. Lewinski AA, Simmons LA. Nurse knowledge and engagement in health policy making: Findings from a pilot study. J Contin Educ Nurs. 2018;49(9):407-15.

27. Maaitah RA, AbuAIRub RF. Exploration of priority actions for strengthening the role of nurses in achieving universal health coverage. Rev Lat Am Enfermagem [Internet]. 2017 [citado 27 de septiembre de 2020];25. Disponible en: https://www.scielo.br/scielo.php?pid=S0104-11692017000100300\&script=sci_arttext

28. SOUZA A, HEINEN H, AMESTOY S, MENDIETA M, PIRIZ M, HECK R. The nurses' work process of Primary Health Care and the National Politics of MedicinalPlants/Phytoterapies. Rev Bras Plantas Med [Internet]. 2016 [citado 4 de 
octubre de 2020];18(2):480-7. Disponible en: https://www.scielo.br/scielo.php?pid=S1516-05722016000200480\&script=sci_arttext 29. dos Santos AR. Educação Permanente em Saúde: construções de enfermeiros da Estratégia Saúde da Família. Rev Baiana Saúde Pública. 2015;38(3):708-24.

30. David HM, Bonetti OP, da Silva MR. Brazilian nursing and the democratization of health: notes on the National Policy of Popular Education in Health. Rev Bras Enferm. 2012;65(1):179-85. 